\title{
Strategic framing to influence clients' risky decisions
}

\author{
Kris De Jaegher ${ }^{1}$ (D) \\ Published online: 2 March 2019 \\ (c) The Author(s) 2019
}

\begin{abstract}
This paper develops a model of persuasive demand inducement in the expert-client relationship. The expert frames the decision on whether or not to buy expert services faced by a client with prospect-theoretic preferences, by making the client see this decision from the perspective of a particular reference point. When inducing a client to buy risky curative services, the expert should set a high reference point, and frame all outcomes as losses. When instead inducing a client to buy safe preventive services, as long as the client's loss aversion is sufficiently high, the expert should set an intermediate reference point, framing high outcomes as gains and low outcomes as losses.
\end{abstract}

Keywords Expert-client relationship - Demand inducement · Prospect theory · Reference points · Framing

\section{Introduction}

In health economics, a large empirical literature looks at whether physicians induce patients to buy unnecessary treatments, specifically when physicians' incomes are under pressure (the so-called supplier-induced demand hypothesis; for an overview, see Peacock and Richardson 2007). The underlying reasoning is that the physician can persuade the patient by changing his preferences and shifting his demand (Evans 1974), where this reasoning may extend to any expert-client relationship. Yet, a model in line with this reasoning is so far missing.

To construct such a model, we draw inspiration from the large empirical literature showing the existence of framing effects (see, e.g., the meta-analysis by Kühberger

Kris De Jaegher

k.dejaegher@uu.nl

1 Utrecht University School of Economics, Utrecht University, Kriekenpitplein 21-22, 3584

EC Utrecht, The Netherlands 
1998), ${ }^{1}$ and apply prospect theory (Kahneman and Tversky 1979; Tversky and Kahneman 1981). In prospect theory, decision-makers have a reference point with respect to which they perceive gains and losses. They are risk averse when considering gains, and risk loving considering losses; moreover, decision-makers are loss averse in caring more about losses than about equally sized gains. If a client has prospect-theoretic preferences, an expert who tries to persuade this client to buy services may frame the client's decision problem by setting a reference point that maximizes the probability that the client buys the services. In particular, consider first the case where buying the services is risky, and not buying the services is safe. Such a scenario may arise if the services recommended by the expert consist of a risky cure, where the client who does not buy the services is not cured and obtains a low outcome with certainty, and the client who does buy the services is cured with probability less than one, and faces the possibility of undergoing an expensive cure that does not work. Given that clients are risk loving for losses, it now seems optimal for the expert to set a high reference point. Yet, the expert's optimal reference point also depends on how risk lovingness changes along the client's valuation function in the loss region, so that a detailed analysis is required.

Second, consider the case where buying the services is safe, and not buying the services is risky. This scenario may be relevant if the expert's services take the form of a preventive treatment, where the client who buys the services avoids all future losses with certainty, but where it is possible that the client would not have incurred any loss without buying the preventive treatment. It is now ambiguous whether the expert should set a low, or an intermediate reference point. The fact that the client is risk averse for gains suggests setting a low reference point, so that all outcomes are seen as gains [this is reflected in the simple reasoning in both Rothman and Salovey (1997) and Rothman et al. (2006) that low reference points should be set to induce risk decisions, and high reference points to induce safe decisions]. Yet, at the same time, loss aversion creates a kink in the client's valuation function around the reference point, which makes the client risk averse for mixed gains and losses. Indeed, as stressed by Wakker (2010, p. 292), “(...) more than half of the risk aversion empirically observed has nothing to do with utility curvature or with probability weighting, but is generated by loss aversion, the main empirical phenomenon regarding reference dependence". Our analysis identifies circumstances in which experts should set an intermediate reference point, exploiting loss aversion; or should instead set a low reference point, exploiting risk aversion in the gains region (where the optimal reference point further depends on how risk aversion changes along the client's valuation function).

Our behavioral model contrasts with theoretical literature that models the expertclient interaction as an asymmetric information game (see Dulleck and Kerschbamer 2006, for an overview; for similar theoretical models of the physician-patient relationship, see Dranove 1988; Calcott 1999; De Jaegher and Jegers 2001). In this literature, the client's state is observed by the expert and not by the client himself, yet the client is an expected utility maximizer who is as strategically sophisticated as the expert. The

\footnotetext{
1 For recent framing experiments, see Hossain and List 2012; Goerg and Kube 2012; Hong et al. 2015; Armantier and Boly 2015; for framing effects outside of the laboratory, see e.g. Pope and Schweitzer (2011), who show that professional golf players are affected by exogenously given reference points.
} 
expert may recommend a specific action, where the client may follow this advice or not (e.g., Pitchik and Schotter 1987; Pesendorfer and Wolinsky 2003); alternatively, the expert provides information, based on which the client himself decides what action to take (e.g., Kamenica and Gentzkow 2011). The client makes his decision taking into account that, given the expert's preferences, her recommendation or information may be biased, and the client thereby constrains the expert's ability to induce demand. ${ }^{2}$

Yet, given the large behavioral economics literature that shows that decision-makers violate the premises of expected utility maximization, it is questionable whether clients always act as modeled in this theoretical literature on the expert-client relationship; moreover, the mentioned violations may be particularly relevant for clients, because they may only buy expert services infrequently. In line with this, a number of recent papers extend the literature on the expert-client relationship to behavioral and experimental economics. Spiegler (2006) studies a market for quacks with clients that follow non-Bayesian reasoning. Norton and Isaac (2012) show experimentally that clients who are aware of the expert's conflict of interest may be subject to ambiguity aversion. Beck et al. (2013) experimentally study the effect of expert guilt aversion, and Kerschbamer et al. (2017) the effect of expert social preferences. The current paper fits into this behavioral literature, and specifically focuses on persuasion by experts of clients that have prospect-theoretic preferences and are subject to framing effects.

The paper is structured as follows. Section 1 contains the model. In Sect. 2, we derive the expert's optimal reference point depending on the precise form of the client's valuation function (Proposition 1), and provide comparative statics results (Proposition 2). This section includes a broad intuition for these results; detailed intuitions, and the proofs of the propositions, are found in Appendices 1 and 2. We end with a discussion in Sect. 3.

\section{The model}

All the symbols used in our model are listed in Appendix 3. At stage 1, the expert (she) observes the client's (he) status, and tells the client all available information, meaning the available actions, and for each action the corresponding outcomes and their probabilities. Only a risky action and a safe action are available. The safe action always yields outcome $M$. The risky action yields outcome $H$ with probability $p_{H}$, and outcome $L$ with probability $p_{L}$ (with $p_{H}+p_{L}=1$ ), where $H>M>L$; $o\left(p_{L}, p_{H}\right)=p_{L} / p_{H}$ denotes the odds of failure. While the expert by assumption reveals all information ("you can either choose an action which always yields outcome $M$, or a risky action which either yields a low outcome $L$ or a high outcome $H$, with odds of failure $o\left(p_{L}, p_{H}\right)$ "), she can still strategically set the client's reference point $R$, which is defined as a reference outcome level which the client uses to assess

\footnotetext{
2 The literature considering the expert-client relationship as a game of asymmetric information often assumes that expertise is a so-called credence good, with clients unable to observe the quality of expertise even after having experienced it (Darby and Karni 1973; Taylor 1995). For instance, once a tooth is extracted, it is impossible to assess how one would have felt if the tooth had not been extracted. In our framing model, as noted by one of the referees, as clients receive all information, expertise need not be a credence good, but may also be an experience good.
} 
the outcomes as gains or losses. This $R$ can be chosen from an interval [ $R_{\min }, R_{\max }$ ] with $R_{\min }<L$ and $R_{\max }>H$. The client sees outcome $X$ as a gain $(X-R)$ if the outcome $X$ exceeds the reference point $R$, and as a loss $(R-X)$ if the outcome $X$ is exceeded by the reference point $R$. At stage 2, after hearing the expert's information, and given the reference point $R$ set by the expert, the client calculates his expected psychic valuation (defined below, henceforth, in short: expected valuation) for each action, and picks the action with the highest expected valuation. Crucially, by assumption, the client does not consider expected valuations from the perspective of reference points other than $R$. Our client is thus not aware that the expert strategically sets $R$, and that if he would consider the decision problem from the perspective of another reference point, he could come to another decision. It follows that our model of expert-client interaction is not a standard game as considered in game theory, but could be termed a "behavioral game". Such a behavioral game fits the category of extensive form games with possibly unaware players as treated by Halpern and Rêgo (2007), where our client is not aware of his own unawareness about the expert's ability to set different reference points.

We now look at the payoffs. The expert always prefers the client to get services rather than not to get services. This translates into two scenarios for the model. In the preventive scenario, the safe action is interpreted as getting costly services (such as preventive healthcare, defense attorney services, or car maintenance) that prevent the client from incurring a future loss (such as getting ill, getting convicted, having one's car breakdown), and the risky action is interpreted as not getting any services. Not getting services leads to the high outcome $(H)$ when the client does not incur the future loss, as the cost of the services is avoided; when the client does incur the loss, it leads to the low outcome $(L)$. When getting the services, the client never incurs the loss, but pays for the cost of the services, leading to the safe intermediate outcome $(M)$. In the curative scenario, the risky action is interpreted as getting services (such as curative healthcare, litigation attorney services, car repair) and the safe action as not getting services. Not getting services means the client faces a certain loss (such as not being cured, not receiving damages, not having one's car repaired), but avoids the cost of the services, leading to the safe intermediate outcome $(M)$. The costly services either repair the loss of the client, leading to the high outcome $(H)$, or do not repair the loss, leading to the low outcome $(L)$, as the client then still incurs the cost of the services.

We now specify the client's valuation function, in accordance with prospect theory. The client does not directly value the absolute outcomes $L, M$ and $H$, but instead values these outcomes as gains or losses with respect to the reference point $R$, where the client thinks differently about gains and losses. For given $R$, the client's valuation function $f(X)$ of any outcome $X$ takes the following form (Tversky and Kahneman 1992):

$$
\begin{array}{r}
X \geq R: f(X)=v(X-R) \\
X<R: f(X)=-\lambda v(R-X)
\end{array}
$$

with $v^{\prime}>0, v^{\prime \prime}<0, v(0)=0$, and $\lambda>1$. 
The function $v(\cdot)$ is assumed to be increasing $\left(v^{\prime}>0\right)$ and concave $\left(v^{\prime \prime}<0\right)$. Using function $v(\cdot)$ for the gains region and the negative of this function for the loss region, the client is modeled as risk averse for gains and risk loving for losses (the so-called reflection effect), in line with the well-known psychological principle (Weber-Fechner law, see Thaler 1999, p. 185) stating that, formulated in health terms, the difference between a gain (respectively, loss) of 1 year in life expectancy and a gain (loss) of 2 years in life expectancy appears larger than the difference between a gain (loss) of 10 years in life expectancy and a gain (loss) of 11 years in life expectancy. The client's marginal valuation is, therefore, smaller the further away from the reference point (diminishing sensitivity). $\lambda$ is the client's degree of loss aversion; by including in the valuation function a coefficient $\lambda>1$ for losses, one adapts the model to the general observation that decision-makers care more about losses (say, a loss of 1 year in life expectancy) than about equally sized gains (say, a gain of 1 year in life expectancy).

It follows now that the client prefers the risky action if

$$
\begin{aligned}
& p_{L} f(L)+p_{H} f(H) \geq f(M) \\
& \Leftrightarrow \\
& \alpha=\frac{f(H)-f(M)}{f(M)-f(L)} \geq o\left(p_{L}, p_{H}\right)
\end{aligned}
$$

and prefers the safe action when the inequality sign in (2) is reversed. $\alpha$ denotes the benefit of getting the high outcome rather than the intermediate outcome, relative to the benefit of getting the intermediate outcome rather than the low outcome. Given the client's valuation in (1), $\alpha$ is a function of $R$, denoted as $\alpha(R)$. By (2), it follows that for a client with given $o\left(p_{L}, p_{H}\right)$, if such $R$ exist, in the curative (preventive) scenario the expert should choose any $R$ such that $\alpha$ exceeds (does not exceed) $o\left(p_{L}, p_{H}\right)$, implying that for each scenario, a range of reference points is optimal.

To obtain more determinate results, we add to our model the realistic assumption that the expert faces heterogeneous clients, where the expert knows the probability of facing each individual type of client, but does not observe the type of the individual client she is currently facing. In particular, because of individual client characteristics that the client observes but the expert does not, clients may differ according to their odds of failure. For instance, a physician may be able to report how the probability of successful treatment depends on the patient's lifestyle, where the patient can then use his private information on his own lifestyle to infer the odds of failure. Formally, we add a stage 0 to the model, where nature chooses the idiosyncratic part $\varepsilon$ of the client's odds of failure $o\left(p_{L}, p_{H}\right)$, where $o\left(p_{L}, p_{H}\right)=o\left(p_{L}, p_{H}\right)^{*}+\varepsilon$, and where $o\left(p_{L}, p_{H}\right)^{*}$ is the generic part of the client's odds of failure (which is identical for all clients). The expert observes $o\left(p_{L}, p_{H}\right)^{*}$, but the client does not. At the same time, the client observes $\varepsilon$, but the expert does not, though the expert knows how $\varepsilon$ is distributed; the expert can infer from this how $o\left(p_{L}, p_{H}\right)$ is distributed, namely according to some density function $g(\cdot)$ over an interval $\left[o_{\min }, o_{\max }\right]$. At stage 1 , the expert communicates to the client the available actions, and the generic odds of failure $o\left(p_{L}, p_{H}\right)^{*}$. Also, 
the expert sets the client's reference point $R$. At stage 2, the client uses (2) (with $\left.o\left(p_{L}, p_{H}\right)=o\left(p_{L}, p_{H}\right)^{*}+\varepsilon\right)$ to determine whether or not to buy services. ${ }^{3}$

\section{Results}

In the curative scenario, by (2) any client with odds of failure $o$ such that $o \leq \alpha$ chooses the curative services, whereas any client with an $o$ such that $o>\alpha$ does not choose the services. It follows that, to maximize the probability that the client buys the curative services, the expert should set a reference point such that $\alpha$ is maximized, where we denote such a reference point as $R^{C}$. As $\alpha=\frac{f(H)-f(M)}{f(M)-f(L)}$, it is an inverse measure of the extent to which the client's valuation function is concave, and thus is at the same time a measure of risk tolerance. The expert who wants to induce the client to buy risky curative services can thus be seen as maximizing the client's risk tolerance.

In the preventive scenario, by (2) it is instead the case that any client with odds of failure $o$ such that $o \geq \alpha$ chooses the preventive services, whereas any client with an $o$ such $o<\alpha$ does not choose the services. Therefore, to maximize the probability that the client buys the preventive services, the expert should set a reference point such that $\alpha$ is minimized, where we denote this reference point as $R^{P}$. The expert who wants to induce the client to buy the safe preventive services can thus be seen as minimizing the client's risk tolerance.

Proposition 1 (which is verbally stated in the body of the paper, and formally in Appendix 1) focuses on the cases where there is a unique optimal reference point, and derives this reference point for the preventive scenario (part I) and the curative scenario (part II), for valuation functions $v(\cdot)$ with an absolute rate of risk aversion (ARA) that is constant in wealth (CARA) or decreasing in wealth (DARA), and for generic levels of loss aversion. The less plausible case where the absolute rate of risk aversion increases in wealth (IARA) is not considered. Next to points I and II, the proposition first broadly states that the optimal reference point does not exceed the intermediate outcome $M$ in the preventive scenario, and is at least $M$ in the curative scenario. Furthermore, for both scenarios, under points (a), (b), etc., candidate optimal reference points are listed in detail; for each such candidate optimal reference point the conditions are listed under which the optimal reference point is indeed optimal.

We here provide a broad intuition for the results in Proposition 1, with a detailed intuition stated in Appendix 1. As already pointed out, to induce the risky curative (safe preventive) services, the expert should maximize (minimize) the client's risk tolerance. Risk tolerance $\alpha$ as a function of the reference point $R$ is represented in Figs. 1 (CARA) and 2 (DARA), where the solid curve represents the case without loss aversion $(\lambda=1)$, whereas the dashed curve and dash-dotted curves represent low $\left(\lambda_{L}\right)$ and high $\left(\lambda_{H}\right)$ loss aversion. As the client is risk averse (risk loving) for pure

\footnotetext{
3 In prospect theory, decision makers additionally use subjective weights, rather than the objective probabilities, where the subjective weights overweight small probabilities (Kahneman and Tversky 1979). We ignore this feature of prospect theory, as it does not make any difference for our results. Still, an alternative way in which patients may have private information is that physicians do not know to what extent the individual patient overweights small probabilities.
} 


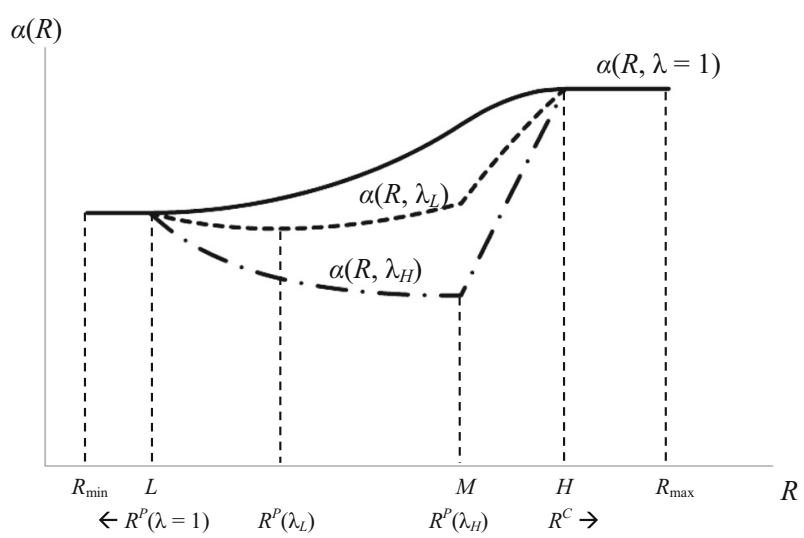

Fig. 1 Risk tolerance $(\alpha)$ as function of client's reference point $(R)$ for CARA valuation function. Solid curve: no loss aversion $(\lambda=1)$; dashed curve: low loss aversion $\left(\lambda_{L}\right)$; dash-dotted curve: high loss aversion $\left(\lambda_{H}\right) . R^{C}\left(R^{P}\right)$ indicates optimal reference point in curative (preventive) scenario

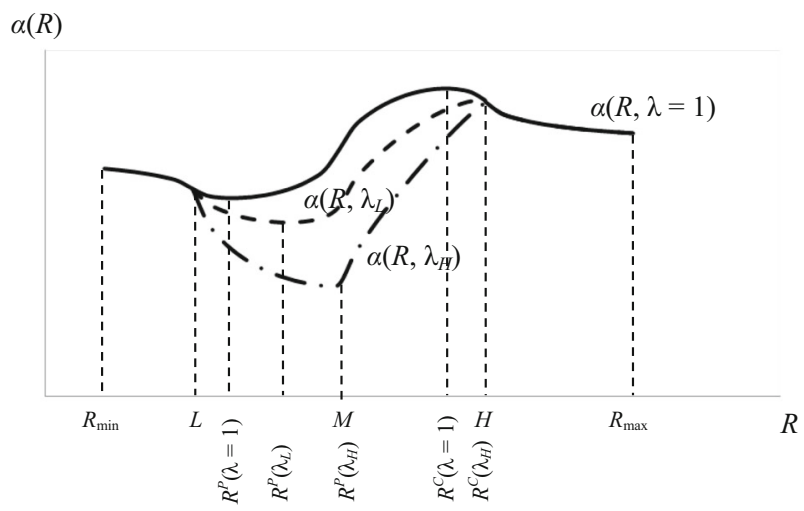

Fig. 2 Idem as Fig. 1, but for DARA valuation function

gains (pure losses), risk tolerance is low (high) for low (high) reference points. For intermediate reference points, where the client perceives mixed gains and losses, he is more risk averse the more loss averse he is, as loss aversion creates a kink in the valuation around the reference point. It follows that, roughly, when inducing the risky curative services the expert should set a high reference point (part II of Proposition 1, where $R^{C}>M$ ), and when inducing the safe preventive services, the expert should set a low (intermediate) reference point for low (high) loss aversion (part I of Proposition 1 , where $R^{P} \leq M$ ). The results in Proposition 1 on the optimal reference points are more specific due to the shape of the valuation function. In the preventive scenario, when loss aversion is low, in spite of the fact that the reference point should be low, it is worthwhile to move slightly into the loss region because, first, with CARA and DARA risk aversion is weakly larger close to the reference point, and second, because the client becomes even more risk averse for mixed gains and losses, due to loss aversion. In the curative scenario, the reference point need not be set higher than $H$, because 
with CARA and DARA risk tolerance is weakly larger close to the reference point. As long as loss aversion is not too large, it is worthwhile to slightly move into the gains region by setting the reference point slightly lower than $H$, to exploit the fact that risk tolerance as at its highest around the reference point. However, if loss aversion is high, the risk aversion that arises for mixed gains and losses means that one should not lower the reference point below $H$.

Proposition 1 Consider a client who takes either a safe action (always yielding an intermediate outcome) or a risky action (yielding either a low or a high outcome). Let the client have prospect-theoretic preferences with an absolute rate of risk aversion that is either constant (CARA) or decreasing in wealth (DARA). Then:

I in the preventive scenario, the safe action means buying preventive services and the risky action means not buying them. The expert sets the reference point $\left(R^{P}\right)$ :

(a) in between the low and intermediate outcome for low loss aversion;

(b) equal to the intermediate outcome for high loss aversion.

II in the curative scenario, the risky action means buying curative services and the safe action means not buying them. The expert sets a reference point $\left(R^{C}\right)$ :

(a) in between the intermediate and high outcome for DARA and low loss aversion;

(b) equal to the high outcome for DARA and high loss aversion;

(c) equal to the high outcome or higher for CARA.

\section{Proof See Appendix 1.}

It is worth to compare the results in Proposition 1 to those of Schwartz et al. (2008) on framing of healthcare decisions without risk. In terms of our model, these authors consider the degenerate case where the "risky" action yields one of the two outcomes, say outcome $H$, with probability 1 . As $H>M$, this means that the client always chooses the "risky" action, whatever $R$. Yet, it still makes sense to see the expert as setting $R$ to either minimize or maximize $f(H)-f(M)$ : when the expert prefers the "risky" (safe) action, she may aim at making its advantage (disadvantage) seem as large (small) as possible. Technically, rather than to maximize or minimize curvature as a function of $R$, Schwartz et al. maximize or minimize the marginal valuation. It is easy to see that the "risky" action feels as advantageous as possible in comparison to the safe action for $R^{C}=H$ in case of high loss aversion, and for a unique $R^{C}$ with $M<R^{C}<H$ in case of low loss aversion (this goes beyond the results of Schwartz et al., who only consider pure gains and pure losses.). This result is in line with Proposition 1 II(a) and II(b) because with DARA, on top of the marginal valuation, also curvature is larger closer to the reference point. Furthermore, the safe action feels the least disadvantageous with respect to the "risky" action when $R^{P}=R_{\min }$, by diminishing sensitivity and by the absence of loss aversion in the gains region. The fact that this result differs from our own results in Proposition 1 I(a) and I(b), shows that having a non-degenerate lottery for the risky action rather than a degenerate lottery, has a clear effect; an intermediate reference point should be chosen to take advantage of the diminishing ARA and/or the fact that there is loss aversion. 
We further explore what the results in Proposition 1 imply for the client's welfare. ${ }^{4}$ Here, we immediately note that, as stressed by Kahneman (1999, pp. 14-15), the valuation function in prospect theory refers to the decision-maker's decision utility, which need not coincide with the decision-maker's experienced utility (see Kahneman et al. 1997). If the expected value of the risky action is lower than or equal to the value of the safe action $\left(p_{L} L+p_{H} H \leq M\right)$, and if we accept that from the perspective of experienced utility, the client is a standard risk-averse expected utility maximizer, then the client's experienced utility is negatively affected by the expert's ability to frame in the curative scenario, but experienced utility is not affected in the preventive scenario. If instead $p_{L} L+p_{H} H>M$, for sufficiently large $p_{H}$, even from the perspective of experienced risk-averse expected utility, the client is still better off with the risky than with the safe action; in this case, the client's experienced utility is not affected by the expert's ability to frame in the curative scenario, but experienced utility is negatively affected in the preventive scenario.

Having derived the optimal reference point in Proposition 1, the logical next step in Proposition 2 is a comparative statics exercise, investigating how the optimal reference point is affected by changes in the parameters, namely by the level of risk aversion, and by the level of loss aversion. Proposition 2 (verbal statement in the body of the paper, precise statement in Appendix 2) separately looks at (I) the preventive scenario and (II) the curative scenario, and for each of these two scenarios in turn separately looks at the comparative statics for (a) the CARA valuation function, and (b) the CRRA valuation function; the focus is on these valuation functions as they have a single parameter for the level of risk aversion, which we can vary.

We again provide a broad intuition for the results in Proposition 2, with a detailed intuition provided in Appendix 2. In the preventive scenario, the expert wants to make the client as risk averse as possible. All else equal a higher level of loss aversion means a higher level of risk aversion around the reference point, giving the expert more incentives to put $R^{P}$ at an intermediate level. At the same time, all else equal, a higher level of risk aversion means that the expert can induce high levels of risk aversion by putting $R^{P}$ low and letting the client perceive gains. In the curative scenario, the expert wants to make the client as risk tolerant as possible. With a CARA valuation function, the client's risk lovingness is constant for any $R$ higher than or equal to the largest outcome, and entering the gains region only decreases risk lovingness. $R^{C}$ is, therefore, unaffected by the parameters. With a CRRA valuation function, the optimal $R^{C}$ is additionally affected by the fact that the level of risk lovingness changes as $R$ is changed, leading to more subtle results, and requiring detailed intuitions (see Appendix 2).

\footnotetext{
4 Azevedo and Gottlieb (2012) show that a risk-neutral firm that can design lotteries and offer those to consumers with prospect-theoretic preferences, under most functional forms suggested in the prospecttheory literature for psychic valuation functions, is able to make infinite profits by offering a lottery that offers an outcome approaching infinity with probability approaching zero. Moreover, high profits can be achieved in a way that is Pareto improving. These problematic implications are not obtained in our model, because our expert is only able to frame an existing lottery, but not to design it. Also, the problem of limitless scope for Pareto improvements is avoided when considering the psychic valuation function as decision utility rather than experienced utility.
} 
Proposition 2 Let a client have prospect-theoretic preferences with either an absolute rate of risk aversion (ARA) that is constant in wealth (CARA) or a relative rate of risk aversion (RRA) that is constant in wealth (CRRA).

I If the expert induces preventive services and sets a reference point $R^{P}$ in between the low and intermediate outcome, then:

(a) with CARA, $R^{P}$ increases in loss aversion and decreases in the ARA;

(b) with CRRA, $R^{P}$ increases in loss aversion and decreases in the RRA.

II If the expert induces curative services (reference point $R^{C}$ ), then:

(a) with CARA, $R^{C}$ is constant in loss aversion and in the ARA;

(b) with CRRA, in case $R^{C}$ lies between the intermediate and the high outcome, $R^{C}$ increases in loss aversion, and $R^{C}$ decreases in the RRA for low RRA and increases in the RRA for high RRA.

Proof See Appendix 2.

\section{Discussion}

As the setting of the model is extremely simple, it is important to check to what extent our simplifying assumptions affect the results. First, in the model the client chooses only between two actions, namely a risky and a safe action, where the risky action has only two possible outcomes. The results are not changed when the client instead decides between a risky and a less risky action, as the same measure of curvature and risk tolerance as in Figs. 1 and 2 can still be employed. When the risky action has more than two outcomes, the qualitative results that the safe action is best induced by framing a low reference point, and that a risky action is best induced by framing a high reference point when loss aversion is low but by framing an intermediate reference point when loss aversion is high, are not changed. Still, in this case the exact level of the optimal reference point is also affected by the probability distribution of the outcomes for the risky action. When the client faces the decision to choose between several treatments and no treatment, where these actions can be ordered according to their riskiness, it may be that the expert can only induce either the safest action, or the riskiest action, but not some action with intermediate riskiness. For instance, if the expert is able to set the reference point such that the client is risk neutral, and if risk neutrality leads the client to be indifferent between all actions, then the expert cannot productively induce treatments with an intermediate level of risk should she prefer to do this, as a client who is induced to be risk averse will choose the safest action, and a client who is induced to be risk loving will choose the riskiest action. This shows that the expert's power to induce specific actions is not unlimited.

Second, we have considered the same valuation function for gains and for losses, with only loss aversion explaining the different assessment of gains and losses. However, literature which estimates the valuation function using the power function (CRRA) often finds the valuation function to be closer to linearity in the loss part than in the gains part (for an overview see Booij et al. 2010). Still, the differences in 
the estimated powers are small, so that the effect on our results is limited. Third, we have assumed that clients value decisions along a single dimension. Yet, clients may consider several dimensions, such as outcome, monetary cost, or unpleasantness of the receiving the services. Separate framing may then take place for each dimension, where framing may be easier for dimensions that are more salient (Rothman et al. 2006). Using the techniques of our analysis, it is possible to extend the model along these lines. Fourth, prospect theory is based on observed decisions over lotteries with monetary outcomes. Yet, prospect-theoretic preferences may have a specific form in specific settings, e.g., for healthcare decisions, preferences may be specific because of the large stakes involved (Attema et al. 2012), and because outcomes are obtained in the distant future (Van der Pol and Ruggeri 2008). Further empirical research is needed here to guide plausible estimates of prospect-theoretic preferences in specific contexts.

Crucial for our results is the assumption that the expert can freely choose any reference point for the client. Yet, the expert could be constrained in what reference point can be suggested in the following ways. First, following Kőszegi and Rabin's (2006), while clients may have prospect-theoretic preferences with respect to a reference point, they may only be willing to consider a reference point that is in line with their expectations, constraining the reference points from which the expert can choose. Thus, a client who expects to buy safe preventive services could then only consider the outcome of buying these services as the reference point. As long as the client is to a sufficient extent loss averse, this happens to also be the optimal reference point for the expert. Yet, a client who expects to buy risky curative services would then in line with Kôszegi and Rabin form a stochastic reference point determined by the expected outcomes of buying the risk curative services.

Second, even if contrary to Kôszegi and Rabin clients would be willing to consider reference points that are not in line with their expectations, following Salant and Siegel (2018), the framing effect of the expert's suggested reference point may wear off if the client gets more time to make his decision, and by talking to family or friends realizes that the decision can also be considered from the perspective of other reference points. In this case, standard models of the expert-client relationship where the client is uninformed but still strategically interacts with experts may be more relevant. Such concerns may explain why the literature on strategic framing is scarce [exceptions are Puppe and Rosenkranz (2011), where manufacturers suggest a retail price, so that buyers' prospect-theoretic preference force retailers to follow this price, and Rosenkranz and Schmitz (2007), where auctioneers may strategically set reserve prices because bidders consider them as reference points].

At the same time, a large empirical literature shows the existence of framing effects (see, e.g., the meta-analysis by Kühberger 1998; for recent framing experiments, see Hossain and List 2012; Goerg and Kube 2012; Hong et al. 2015; Armantier and Boly 2015; for framing effects outside of the laboratory, see, e.g., Pope and Schweitzer (2011), who show that professional golf players are affected by exogenously given reference points). We argue that framing effects may be plausible in the expert-client relationship, because the client may face the modeled decisions infrequently, and may not be able to consult other experts or to talk to other clients who have faced similar decisions. For this reason, even if reference points may in general be determined by 
expectations, the client may find it difficult to form any expectations in this context. Moreover, even if the reference points suggested by experts wear off in the long term, clients may have to make short-term decisions (e.g., a patient needs to decide on the spot whether or not a dentist performs a root-canal treatment), and because of the hot-cold empathy gap (Loewenstein 2005) may not realize that if they had more time to make the decision, they could see the decision problem from the perspective of other reference points than the one suggested by the expert.

We end by noting that standard models of the expert-client relationship where clients are fully rational on the one hand, and our model where experts face no constraints in the extent to which they can frame the clients' decisions on the other hand, may be seen as opposed benchmarks, to which real-world expert-client encounters should be compared. Our theoretical analysis cannot determine how close the realworld expert-client relationship lies to our strategic-framing benchmark, and this question can only be answered by means of empirical analysis. While it seems difficult to catch the expert in the act of inducing demand by means of framing, two types of empirical analyses could still test the predictions of our model. First, our model provides predictions on which types of clients should be more susceptible to framing of particular types of services (curative, or preventive services). While one may not directly be able to observe how risk averse or loss averse clients are, one could fall back on studies that relate risk aversion or loss aversion to more easily observed characteristics such as sex or age, to come to hypotheses on how such personal characteristics make one susceptible to demand inducement in particular contexts, and lead one to consume more services. Second, our predictions could be tested in a well-designed laboratory experiment, involving both participants taking on the role of clients, and the role of experts. We hope that such an experiment is then able to answer the question whether the expert-client relationship is purely a game of strategic information transmission, or whether strategic framing (also) plays a role.

Open Access This article is distributed under the terms of the Creative Commons Attribution 4.0 International License (http://creativecommons.org/licenses/by/4.0/), which permits unrestricted use, distribution, and reproduction in any medium, provided you give appropriate credit to the original author(s) and the source, provide a link to the Creative Commons license, and indicate if changes were made.

\section{Appendix 1}

\section{Detailed intuition of Proposition 1}

As our results depend on insight into how $\alpha(R)$ changes as a function of $R$, we obtain intuitions for our results by explaining separately how the shape of the function $v(\cdot)$, and how the level of loss aversion $\lambda$ impacts the shape of $\alpha(R)$.

We first look at how the shape of $v(\cdot)$ affects $\alpha(R)$. Risk tolerance is the opposite of risk aversion, and a standard measure of the latter is the ARA. As an increase in the client's reference point is the analog of a decrease in wealth, the shape of $\alpha(R)$ at first sight depends on how ARA changes as wealth changes. In the pure gains and pure loss region, this is indeed the case. With CARA, $\alpha$ is low and flat for $R \leq L$ 
and high and flat for $R \geq H$ (Fig. 1). With DARA (Fig. 2), as an increase in $R$ is the analog of a decrease in wealth, and as in the gains region a decrease in wealth means higher risk aversion and, therefore, lower risk tolerance, $\alpha(R)$ decreases in $R$ for $R \leq L .{ }^{5}$ Because of the symmetry imposed by the reflection effect, in the loss region an increase in wealth means higher risk tolerance, so that $\alpha(R)$ also decreases in $R$ for $R \geq H$.

Strictly speaking, the ARA is a measure of local risk aversion, defined for infinitesimal gambles. While our risky action is not an infinitesimal gamble ( $L$ and $H$ are not arbitrarily close to $M$ ), in the pure gains and pure loss region, results still depend only on whether we have DARA or CARA. For $R$ such that the client perceives both gains and losses, however, we need a measure of global risk aversion. An inverse measure of global risk aversion is found precisely in $\alpha$, as it is an inverse measure of global concavity. Leaving out loss aversion for the moment and focusing purely on the reflection effect, the following is now clear. First, the slope of $\alpha(R)$ just above $L$ is the same as just below $L$; the same applies for the slopes around $M$ and $H$. Furthermore, an $R$ between $L$ and $H$ exists such that the client is globally risk neutral, meaning that while $\alpha$ is low (high) for $R \leq L(R \geq H)$, it is intermediate for intermediate $R$. Finally, $\alpha(R)$ is increasing in $R$ approaching $R=M$ from the right. To see why, note that for $R=M$, a slight increase in $R$ has two effects. Because of diminishing sensitivity, first, the difference in the valuation of $M$ and $L$ becomes smaller. Second, for the same reason, the difference in the valuation of $H$ and $M$ becomes larger; this effect is further reinforced because for the valuation of $M$, the convex region of the valuation function is entered.

For CARA, in the absence of loss aversion, these intuitions suggest a monotonic increase from low to high risk tolerance in the region of mixed gains and losses (solid curves in Fig. 1). It follows that with CARA the expert should set $R^{P}\left(R^{C}\right)$ in any way such that $R^{P} \leq L\left(R^{C} \geq H\right)$. For DARA, the picture is more complex. By the previous, $\alpha$ is now low and decreasing in $R$ around $L$, is intermediate and increasing in $R$ around $M$, and is high but decreasing in $R$ around $H$. This suggests that for DARA, in the absence of loss aversion, $\alpha(R)$ takes the form of the solid curve in Fig. 2, with $L<R^{P}<M$ and $M<R^{C}<H$. As these results apply without loss aversion, it is intuitive that similar results apply for low loss aversion, which explains in Proposition 1 cases I(a) and II(a) for DARA (dashed curves in Figs. 1, 2).

When adding loss aversion to the analysis, the following is clear. First, loss aversion does not impact $\alpha(R)$ in the pure gains or pure loss region, because it only has impact for mixed gains and losses. Thus, in Figs. 1 and 2 , for $R \leq L$ and $R \geq H$ the curves with different levels of loss aversion coincide. Second, as we let loss aversion become very high, global risk tolerance at $R=M$ becomes minimal, as the kink in the valuation function around the reference point becomes extreme. It follows that as

\footnotetext{
5 In a different context (students' choice for a risky or safe education as a function of their reference points), Page (2005) in his Fig. 2 plots the difference in expected utility of a risky gamble and a safe gamble as a function of the reference point, where a DARA utility function is assumed. Figures 1 and 2 instead plot, as a function of the reference point, the incremental utility of having the high outcome of a risky gamble rather than the intermediate outcome of a safe gamble, relative to the incremental utility of having the intermediate outcome of the safe gamble rather than the low outcome of a risky gamble. Because a higher value of our $\alpha$ implies that the risky gamble should also be more attractive relative to the safe gamble, our Figs. 1 and 2 and Page's Fig. 2 have a similar shape.
} 
long as loss aversion is sufficiently high, $R^{P}=M$ [see Proposition $\left.1 \mathrm{I}(\mathrm{b})\right]$. Third, the slope of $\alpha(R)$ approaching $M$ from the right continues to be positive when there is loss aversion. Loss aversion has the additional effect that the difference between the valuation of $H$ and $M$ is further increased as the loss region is entered.

Fourth, the kink in the valuation function around the reference point translates into a kink in $\alpha$ for $R$ around $L, M$ and $H$. Starting at $R=L$, as $R$ is increased and the loss region is entered, the difference in the valuation of $M$ and $L$ increases because of loss aversion. By (2), it follows that the slope of $\alpha$ is smaller approaching $L$ from the right than from the left. This means that at $R=L, \alpha(R)$ never has a peak for CARA or DARA. Starting at $R=M$ and lowering $R$, the presence of loss aversion decreases the difference in valuation between $M$ and $L$ as one partially enters the gains region. For sufficiently high loss aversion, this has a large impact on the denominator of $\alpha$ [see (2)], so that $\alpha$ decreases in $R$ approaching $M$ from the left, and has a peak at $R=M$. Finally, starting at $R=H$ and lowering $R$, the presence of loss aversion decreases the difference in valuation of $H$ and $M$. For sufficiently high loss aversion, this has a large impact on the numerator of $\alpha$ [see (2)]. It follows that loss aversion makes the slope of $\alpha$ larger approaching $H$ from the right than from the left. This means that at $R=H, \alpha(R)$ never has a peak for CARA, but does have a peak for DARA when loss aversion is sufficiently high.

All results in Proposition 1 are now readily obtained conditional on the sign of the slope of $\alpha(R)$ changing at most once in each of the ranges $L<R<M$ and $M<R<H$. It can be checked that for $v(\cdot)$ with the CARA form, this condition is always valid. For $v(\cdot)$ with the DARA form, the condition can also be checked to be valid for the specific case where $v(\cdot)$ has the CRRA (constant relative rate of risk aversion) form. Proposition 1 provides more general results for $v(\cdot)$ with the DARA form, conditional on these $v(\cdot)$ also being well behaved, in the following sense: with DARA, as we increase $R$ starting from $R$ below $L$, because of the DARA form global risk tolerance first decreases, then all else equal increases because of the effect of loss aversion that plays in the region of mixed gains and losses, and finally decreases again close to the pure loss region because of the DARA form. With well-behaved $v(\cdot)$ (i.e., the slope of $\alpha(R)$ changes sign at most once in each of the ranges $L<R<M$ and $M<R<H$ ), the combination of these effects leads first to a decrease in global risk aversion, then again to an increase, and finally again to a decrease, without any further sign changes of the slope. The fact that $v(\cdot)$ of the CRRA form are well behaved, suggests that limiting oneself to $v(\cdot)$ with the DARA form that are well behaved as well, is not very restrictive.

The results obtained are the following. In the preventive scenario, for both CARA and DARA, $R^{P}>L$; when loss aversion is sufficiently high, $R^{P}=M$, otherwise it lies between $L$ and $M$ (where it should be noted that as shown in Appendix 1, when $v^{\prime}(0)$ approaches infinity, only loss aversion approaching infinity is sufficiently high to make $R^{P}$ approach $M$ ). In the curative scenario, for CARA, whatever the level of loss aversion, $R^{C}$ can be put anywhere equal to or larger than $H$. For well-behaved DARA, $R^{C}$ is equal to $H$ for sufficiently high loss aversion, and lies in between $M$ and $H$ otherwise (where a similar remark applies about $v^{\prime}(0)$ approaching infinity, and what defines sufficiently high loss aversion). 


\section{Formal statement and proof of Proposition 1}

Proposition 1 Consider valuation functions $v(\cdot)$ of the CARA or DARA form. For the $D A R A$ case, consider well-behaved valuation functions, where the sign of the slope of $\alpha(R)$ changes at most once in both the range $L<R<M$ and the range $M<R<H$. Then:

I. In the preventive scenario, assuming $o_{\min }<\alpha\left(R^{P}\right) \leq o_{\max }$, it is the case that $R^{P} \leq M$, and in particular

(a) $R^{P}$ is a unique value with $L<R^{P}<M$ conditional on sufficiently low loss aversion;

(b) $R^{P}=M$ conditional on sufficiently high loss aversion.

II. In the curative scenario, assuming $o_{\min } \leq \alpha\left(R^{C}\right)<o_{\max }$, it is the case that $R^{C}>M$, and in particular:

(a) $R^{C}$ is a unique value with $M<R^{C}<H$ conditional on (1) sufficiently low loss aversion, and (2) a $v(\cdot)$ with the DARA form;

(b) $R^{C}=H$ conditional on (1) sufficiently high loss aversion, and (2) a $v(\cdot)$ with the DARA form;

(c) $R^{C}$ is any value such that $H \leq R^{C} \leq R_{\max }$ conditional on a $v(\cdot)$ with the CARA form.

Proof We focus on cases where a unique reference point is optimal. In the preventive scenario, this occurs when $o_{\min }<\alpha\left(R^{P}\right) \leq o_{\max }$. When instead $o_{\min } \geq \alpha\left(R^{P}\right)$, any reference point $R$ such that $o_{\min } \geq \alpha(R)$ ensures that the client always buys preventive services; when $\alpha\left(R^{P}\right)>o_{\max }$, the client will not buy preventive services, whatever the reference point the expert sets. In the curative scenario, there is a unique reference point when $o_{\min } \leq \alpha\left(R^{C}\right)<o_{\max }$, When instead $o_{\min }>\alpha\left(R^{C}\right)$, the client will not buy curative services, whatever the reference point; when $\alpha\left(R^{C}\right) \geq o_{\max }$, any reference point $R$ such that $\alpha(R) \geq o_{\max }$ ensures that the client always buys curative services.

Using the psychic valuation function in (2), we can see that as a function of the relation between $R$ and the outcomes, the expression for $\alpha$ can take on four possible forms:

$$
\begin{gathered}
\alpha(R \leq L)=\frac{v(H-R)-v(M-R)}{v(M-R)-v(L-R)} \\
\alpha(L<R \leq M)=\frac{v(H-R)-v(M-R)}{v(M-R)+\lambda v(R-L)} \\
\alpha(M<R \leq H)=\frac{v(H-R)+\lambda v(R-M)}{\lambda v(R-L)-\lambda v(R-M)} \\
\alpha(R>H)=\frac{v(R-M)-v(R-H)}{v(R-L)-v(R-M)}
\end{gathered}
$$


Step 1. Let us first compare all the cases where $R$ is equal to exactly one of the values $L, M, H$ :

$$
\begin{gathered}
\alpha(R=L)=\frac{v(H-L)-v(M-L)}{v(M-L)} \\
\alpha(R=M)=\frac{v(H-M)}{\lambda v(M-L)} \\
\alpha(R=H)=\frac{v(H-M)}{v(H-L)-v(H-M)}
\end{gathered}
$$

It is clear from (7) to (9) that for $\lambda=1$, given diminishing sensitivity, $v(H-L)-v(M-L)<v(H-M)$ and $v(H-L)-v(H-M)<v(M-L)$, so that $\alpha(R=L)<\alpha(R=M)<\alpha(R=H)$. The same result is obtained as long as $\lambda<$ $\frac{v(H-H)}{v(H-L)-v(M-L)}=\lambda^{*}$ (note that $\lambda^{*}$ is larger than 1 as soon as there is diminishing sensitivity). If $\lambda>\lambda^{*}$, then $\alpha(R=M)<\alpha(R=L)<\alpha(R=H)$.

We next check how expressions (3)-(6) change as a function of $R$, where we pay particular attention to the cases where $R$ lies close to one of the values $L, M, H$. In Step 2, we start with the cases $R \leq L$ and $R>H$.

Step 2.

$$
\begin{aligned}
\frac{\partial \alpha(R \leq L)}{\partial R}=- & \frac{\left[v^{\prime}(H-R)-v^{\prime}(M-R)\right][v(M-R)-v(L-R)]}{[v(M-R)-v(L-R)]^{2}} \\
& +\frac{[v(H-R)-v(M-R)]\left[v^{\prime}(M-R)-v^{\prime}(L-R)\right]}{[v(M-R)-v(L-R)]^{2}}
\end{aligned}
$$

It follows that the sign of (10) depends on the sign of

$$
\frac{\left|v^{\prime}(H-R)-v^{\prime}(M-R)\right|}{v(H-R)-v(M-R)}-\frac{\left|v^{\prime}(M-R)-v^{\prime}(L-R)\right|}{v(M-R)-v(L-R)}
$$

Furthermore:

$$
\frac{\partial \alpha(R>H)}{\partial R}=\frac{\left[v^{\prime}(R-M)-v^{\prime}(R-H)\right][v(R-L)-v(R-M)]-}{[v(R-M)-v(R-H)]\left[v^{\prime}(R-L)-v^{\prime}(R-M)\right]}
$$

It follows that the sign of (12) depends on the sign of

$$
-\frac{\left|v^{\prime}(R-H)-v^{\prime}(R-M)\right|}{v(R-M)-v(R-H)}+\frac{\left|v^{\prime}(R-M)-v^{\prime}(R-L)\right|}{v(R-L)-v(R-M)}
$$

Each of the four terms in (11) and (13) measures the slope of the secant through two distinct points on this same function, and, therefore, measure average ARA between 
these two points. It immediately follows that if it is everywhere the case that ARA increases, decreases or remains constant for higher outcomes (and, therefore, also for higher utility), then average ARA as reflected in these four terms will also increase, decrease or remain constant. We, therefore, obtain the following cases:

1. With CARA, $\frac{\partial \alpha(R \leq L)}{\partial R}=\frac{\partial \alpha(R>H)}{\partial R}=0$.

2. With DARA, $\frac{\partial \alpha(R \leq L)}{\partial R}<0$ and $\frac{\partial \alpha(R>H)}{\partial R}<0$.

We further have

$$
\begin{aligned}
\frac{\partial \alpha(L<R \leq M)}{\partial R}= & \frac{\left[-v^{\prime}(H-R)+v^{\prime}(M-R)\right][v(M-R)+\lambda v(R-L)]-}{[v(H-R)-v(M-R)]\left[-v^{\prime}(M-R)+\lambda v^{\prime}(R-L)\right]} \\
\left.\frac{\partial \alpha(M-R)+\lambda v(R-L)]^{2}}{\partial R}=R \leq H\right) & \frac{\left[\lambda v^{\prime}(R-M)-v^{\prime}(H-R)\right][\lambda v(R-L)-\lambda v(R-M)]-}{[\lambda v(R-M)+v(H-R)]\left[\lambda v^{\prime}(R-L)-\lambda v^{\prime}(R-M]\right.}
\end{aligned}
$$

In the following steps, we use (14) and (15) to derive further results about the shape of $\alpha$ as a function of $R$.

Step 3. Evaluating (10) and (14) around $R=L$, we obtain

$$
\left.\frac{\partial \alpha(R \leq L)}{\partial R}\right|_{R=L}=\frac{\begin{array}{c}
-\left[v^{\prime}(H-L)-v^{\prime}(M-L)\right] v(M-L)+ \\
{\left[v(H-L)-v(M-L]\left[v^{\prime}(M-L)-v^{\prime}(0)\right]\right.}
\end{array}}{v(M-R)^{2}}
$$

and

$$
\begin{aligned}
& -\left[v^{\prime}(H-L)-v^{\prime}(M-L)\right] v(M-L)+ \\
& \left.\frac{\partial \alpha(L<R \leq M)}{\partial R}\right|_{R \rightarrow L}=\frac{\left[v(H-L)-v(M-L]\left[v^{\prime}(M-L)-\lambda v^{\prime}(0)\right]\right.}{v(M-R)^{2}}
\end{aligned}
$$

It follows that

$$
\left.\frac{\partial \alpha(R \leq L)}{\partial R}\right|_{R=L}>\left.\frac{\partial \alpha(L<R \leq M)}{\partial R}\right|_{R \rightarrow L}
$$

The following, therefore, applies:

1. With CARA, by (18) and Step 2(1), $\left.\frac{\partial \alpha(L<R \leq M)}{\partial R}\right|_{R \rightarrow L}<0=\left.\frac{\partial \alpha(R \leq L)}{\partial R}\right|_{R=L}$.

2. With DARA, by (18) and Step 2(2), $0>\left.\frac{\partial \alpha(R \leq L)}{\partial R}\right|_{R=L}>\left.\frac{\partial \alpha(L<R \leq M)}{\partial R}\right|_{R \rightarrow L}$. 
Step 4. Evaluating (14) and (15) around $R=M$, we obtain

$$
\begin{aligned}
& \left.\frac{\partial \alpha(L<R \leq M)}{\partial R}\right|_{R=M} \\
& =\frac{\left[v^{\prime}(0)-v^{\prime}(H-M)\right] \lambda v(M-L)-v(H-M)\left[\lambda v^{\prime}(M-L)-v^{\prime}(0)\right]}{[\lambda v(M-L)]^{2}}
\end{aligned}
$$

and

$$
\begin{aligned}
& \left.\frac{\partial \alpha(M<R \leq H)}{\partial R}\right|_{R \rightarrow M} \\
& =\frac{\left[\lambda v^{\prime}(0)-v^{\prime}(H-M)\right] \lambda v(M-L)-v(H-M)\left[\lambda v^{\prime}(M-L)-\lambda v^{\prime}(0)\right]}{[\lambda v(M-L)]^{2}}
\end{aligned}
$$

Given diminishing sensitivity, it follows by (20) that it is always the case that $\left.\frac{\partial \alpha(M<R \leq H)}{\partial R}\right|_{R \rightarrow M}>0$. (19) and (20) imply that $\left.\frac{\partial \alpha(M<R \leq H)}{\partial R}\right|_{R \rightarrow M}>$ $\left.\frac{\partial \alpha(L<R \leq M)}{\partial R}\right|_{R=M}$. Furthermore, by (19), $\left.\frac{\partial \alpha(L<R \leq M)}{\partial R}\right|_{R=M}>0$ if $v^{\prime}(0)>$ $\left[v^{\prime}(M-L) v(H-M)+v^{\prime}(H-M) v(M-L)\right] / v(M-L)$. For $v^{\prime}(0)<$
$\left[v^{\prime}(M-L) v(H-M)+v^{\prime}(H-M) v(M-L)\right] / v(M-L),\left.\frac{\partial \alpha(L<R \leq M)}{\partial R}\right|_{R=M}>0$ if $\lambda<\lambda_{M}$, and $\left.\frac{\partial \alpha(L<R \leq M)}{\partial R}\right|_{R=M}<0$ if $\lambda>\lambda_{M}$, with

$$
\lambda_{M}=\frac{v^{\prime}(0) v(H-M)}{v^{\prime}(M-L) v(H-M)-\left[v^{\prime}(0)-v^{\prime}(H-M)\right] v(M-L)}
$$

where it can be checked that it is always valid that $\lambda_{M}>1$. It should be noted that when $v^{\prime}(0)$ approaches infinity, as is the case for, e.g., the CRRA utility function, by (21), $\lambda_{M}$ approaches infinity is as well.

Step 5. Evaluating (12) and (15) around $R=H$, we obtain

$$
\begin{gathered}
\left.\frac{\partial \alpha(M<R \leq H)}{\partial R}\right|_{R=H}=\frac{\left[\lambda v^{\prime}(H-M)-v^{\prime}(0)\right][\lambda v(H-L)-\lambda v(H-M)]-}{\lambda v(H-M)\left[\lambda v^{\prime}(H-L)-\lambda v^{\prime}(H-M)\right]} \\
\frac{\partial \alpha v(H-L)-\lambda v(H-M)]^{2}}{\left[v^{\prime}(H-M)-v^{\prime}(0)\right][v(H-L)-v(H-M)]-} \\
=\frac{v(H-M)\left[v^{\prime}(H-L)-v^{\prime}(H-M)\right]}{[v(H-L)-v(H-M)]^{2}}
\end{gathered}
$$


It follows from (22) to (23) that

$$
\left.\frac{\partial \alpha(R>H)}{\partial R}\right|_{R \rightarrow H}<\left.\frac{\partial \alpha(M<R \leq H)}{\partial R}\right|_{R=H}
$$

The following now applies:

1. For CARA, by (24) and Step 2(1), $\left.\frac{\partial \alpha(R>H)}{\partial R}\right|_{R \rightarrow H}=0<\left.\frac{\partial \alpha(M<R \leq H)}{\partial R}\right|_{R=H}$.

2. For DARA, by (24) and Step 2(2), $\left.\frac{\partial \alpha(R>H)}{\partial R}\right|_{R \rightarrow H}<\left.\frac{\partial \alpha(M<R \leq H)}{\partial R}\right|_{R=H} ^{R=H}$ and $\left.\frac{\partial \alpha(R>H)}{\partial R}\right|_{R \rightarrow H}<0$. In this case, $\left.\frac{\partial \alpha(M<R \leq H)}{\partial R}\right|_{R=H}<0$ if $\lambda<\lambda_{H}$, and $\left.\frac{\partial \alpha(M<R \leq H)}{\partial R}\right|_{R=H}>0$ if $\lambda>\lambda_{H}$, with

$$
\lambda_{H}=\frac{v^{\prime}(0)[v(H-L)-v(H-M)]}{\left[v^{\prime}(H-M) v(H-L)-v^{\prime}(H-L) v(H-M)\right]}
$$

where it can be checked that for DARA, $\lambda_{H}>1$. We note again that when $v^{\prime}(0)$ approaches infinity, as is the case for, e.g., the CRRA utility function, by (25), $\lambda_{H}$ approaches infinity is as well.

Step 6. In this final step, we put all the results of the previous steps together.

1. For CARA, by Step 2, $\alpha(R)$ is flat in $R$ both for $L \leq R$ and $R \geq H$, where by diminishing sensitivity $\alpha(R)$ is higher for $H \geq R$. By Steps 4 and 5, $\alpha(R)$ increases in $R$ just above $M$ and just below $H$, so that given the fact that for CARA the slope of $\alpha(R)$ changes sign at most once between $M$ and $H, \alpha(R)$ increases everywhere in $R$ for $M<R<H$. By Step 3, $\alpha(R)$ decreases in $R$ just above $L$. By Step 4, if $v^{\prime}(0)>\left[v^{\prime}(M-L) v(H-M)+v^{\prime}(H-M) v(M-L)\right] / v(M-L), \alpha(R)$ decreases in $R$ just below $M$. By the assumption that the slope of $\alpha(R)$ changes sign at most once between $L$ and $M$, it follows that $\alpha(R)$ decreases in $R$ for $L<R<M$. Yet, by (8) in Step 1, as loss aversion approaches infinity, $\alpha(M)$ approaches zero, which is the lowest value that $\alpha(R)$ can reach. In this sense, the claim that $R^{P}=M$ for sufficiently large loss aversion applies in this case, where a sufficiently large level of loss aversion is one approaching infinity. If $v^{\prime}(0)<$ $\left[v^{\prime}(M-L) v(H-M)+v^{\prime}(H-M) v(M-L)\right] / v(M-L), \alpha(R)$ decreases in $R$ just below $M$ if $\lambda<\lambda_{M}$, and $\alpha(R)$ increases in $R$ just below $M$ if $\lambda>\lambda_{M}$. By the assumption that the slope of $\alpha(R)$ changes sign at most once, in the former case, $\alpha(R)$ has a local minimum for $R=M$; in the latter case, $\alpha(R)$ reaches a local minimum for $L<R<M$. It follows that $R^{C}$ is any value of at least $H$. $R^{P}$ lies strictly between $L$ and $M$ for sufficiently low loss aversion, and lies precisely at $M$ for sufficiently high loss aversion.

2. For DARA, by Step 2, $\alpha(R)$ is decreasing in $R$ both for $L \leq R$ and $R \geq H$, where by diminishing sensitivity $\alpha(R)$ is everywhere higher for $H \geq R$. For $L<R<M$, by Steps 3 and 4, the analysis is analogous to CARA. By Step 4, $\alpha(R)$ increases in $R$ just above $M$. By Step $5, \alpha(R)$ decreases in $R$ just below $H$ if $\lambda<\lambda_{H}$, and increases in $R$ just below $H$ if $\lambda>\lambda_{H}$; by the assumption 
that the slope of $\alpha(R)$ changes sign at most once, $\alpha(R)$ reaches a local maximum for $M<R<H$ in the former case, and has a local maximum for $R=H$ in the latter case. It follows that $R^{C}$ lies strictly between $M$ and $H$ for sufficiently low loss aversion, and precisely at $H$ for sufficiently high loss aversion. $R^{P}$ lies strictly between $L$ and $M$ for sufficiently low loss aversion, and precisely at $M$ for sufficiently high loss aversion. It should be noted that when $v^{\prime}(0)$ approaches infinity, such as is the case for the CRRA utility function, the critical level of loss aversion for which $\alpha(R)$ has a peak at $M$ and $H$, approaches infinity itself.

\section{Appendix 2}

\section{Detailed intuition of Proposition 2}

Consider first the preventive scenario, and start at $R=L$. As any CRRA valuation function has the DARA form, this means that for higher $R$, the client becomes more risk averse. This gives the expert an incentive to partially enter the loss region, as risk aversion will then continue to increase for the payoffs that remain in the gains region. All else equal, higher loss aversion increases this incentive, as the difference in valuation between $M$ and $L$ increases. At the same time, all else equal, higher RRA means that both ARA ( $=\mathrm{RRA} / Y$, where $Y$ is either a gain or a loss) and absolute risk lovingness increase just above and just below the reference point, giving the expert less reason to enter the loss region. With a CARA valuation function, as risk aversion is not affected by $R$, in the absence of loss aversion, there is no incentive to enter the loss region, but loss aversion again increases this incentive. The intuition for the effect of an increase in ARA is the same as for an increase in RRA.

Consider next the curative scenario, and start at $R=H$. Given that the CRRA valuation function has the DARA form, in the loss region lower $R$ means higher risk lovingness, giving the expert an incentive to slightly enter the gains region. However, the higher loss aversion, the more entering the gains region causes risk aversion, thus implying that the expert becomes less inclined to lower $R$. For the same reason, under CARA there is no reason at all to enter the loss region, and $R^{C}$ is unaffected by higher loss aversion.

Let us now look at the effect of ARA and RRA on $R^{C}$. With CARA, risk aversion is not affected by $R$ in the pure loss region; because of loss aversion, risk aversion is positively affected when the gains region is entered. It follows that there is no reason to enter the gains region, whatever the level of ARA, meaning that $R^{C}$ is unaffected by ARA. For CRRA, take as a starting point a case with intermediate RRA, and with loss aversion low. Given the DARA form of CRRA, in the pure loss region, risk lovingness increases as $R$ is decreased; this effect continues to play as the gains region is partially entered, as risk lovingness continues to increase for the payoffs that remain in the loss region. The fact that loss aversion is low means that the fact that risk aversion may be high for mixed gains and losses, plays less of a role. It follows that for intermediate RRA and low loss aversion, $M<R^{C}<H$. Compare this, first, to the limit case where $v(\cdot)$ approaches a linear function (RRA approaches zero). Then only the effect of loss aversion is at work, and the expert wants to avoid the client perceiving any gains, as 
this reduces risk tolerance, so that $R^{C}=H$. As a decrease in RRA (compared to the case of an intermediate RRA) means an increase in $R^{C}$, this means that $R^{C}$ decreases in RRA. Second, compare to the limit case where RRA becomes extremely high. Then by the fact that ARA $=\mathrm{RRA} / Y$ (where $Y$ is either a gain or a loss), risk aversion is high just above the reference point. Again, the expert wants to avoid the client perceiving any gains, and sets $R^{C}=H$. As this is the result of an increase in RRA (compared to the case of an intermediate RRA), it follows that $R^{C}$ increases in RRA. This intuition explains the ambiguous result in Proposition 2 II(b).

\section{Formal statement and proof of Proposition 2}

\section{Proposition 2}

I. Consider the preventive scenario, and let there be a unique $R^{P}$ with $L<R^{P}<M$. Then

(a) under CARA, $\partial R^{P} / \partial \lambda>0$ and $\partial R^{P} / \partial \mathrm{ARA}<0$;

(b) under CRRA, $\partial R^{P} / \partial \lambda>0$, and $\partial R^{P} / \partial \mathrm{RRA}<0$.

II. Consider the curative scenario. Then

(a) under CARA, $\partial R^{C} / \partial \lambda=0$, and $\partial R^{C} / \partial \mathrm{ARA}=0$;

(b) under CRRA, in case there is a unique $R^{C}$ with $M<R^{C}<H$, we have $\partial R^{C} / \partial \lambda>0$, and $\partial R^{C} / \partial \mathrm{RRA}<0$ for low RRA, but $\partial R^{C} / \partial \mathrm{RRA}>0$ for high RRA.

Proof: Steps 1 and 2 look at the preventive scenario, respectively, for CARA and CRRA, Steps 3 and 4 do the same for the curative scenario.

Step 1. Consider the generic CARA valuation function $v(X)=1-\exp [-\mathrm{ARA} \times$ $X]$. Then it is easily checked, by putting the RHS of (14) equal to zero, that $R^{P}=$ $L-\frac{1}{\text { ARA }} \ln \frac{1+\lambda}{2 \lambda}$, so that $\partial R^{P} / \partial \lambda>0$, and for $\lambda>1$, we have $\partial R^{P} / \partial \mathrm{ARA}<0$.

Step 2. The sign of $\partial R^{P} / \partial \lambda$ can be found by taking the total differential of $\left.\frac{\partial \alpha(L<R \leq M)}{\partial R}\right|_{R=R^{P}}=0$ with respect to $R^{P}$ and $\lambda$, and equating to zero, or

$$
\left.\frac{\partial^{2} \alpha(L<R \leq M)}{\partial R^{2}}\right|_{R=R^{P}} \mathrm{~d} R^{P}+\left.\frac{\partial^{2} \alpha(L<R \leq M)}{\partial R \partial \lambda}\right|_{R=R^{P}} \mathrm{~d} \lambda=0
$$

In the same way, the sign of $\partial R^{P} / \partial \mathrm{ARA}$ can be found by taking the total differential of $\left.\frac{\partial \alpha(L<R \leq M)}{\partial R}\right|_{R=R^{P}}=0$ with respect to $R^{P}$ and RRA, and equating to zero, or

$$
\left.\frac{\partial^{2} \alpha(L<R \leq M)}{\partial R^{2}}\right|_{R=R^{P}} \mathrm{~d} R^{P}+\left.\frac{\partial^{2} \alpha(L<R \leq M)}{\partial R \partial \mathrm{RRA}}\right|_{R=R^{P}} \mathrm{dRRA}=0
$$

Looking at (14), one obtains $\left.\frac{\partial \alpha(L<R \leq M)}{\partial R}\right|_{R=R^{P}}=0$ when the numerator of the RHS of (14) equals zero, a condition which under the generic CRRA valuation function $v$ $(X)=X^{1-R R A}$ can be written as

$$
(1-\mathrm{RRA})\left(H-R^{P}\right)^{-\mathrm{RRA}} \lambda\left(R^{P}-L\right)^{-\mathrm{RRA}}(H-L)\left\{X^{P}\right\}=0,
$$


where $X^{P}=\left(\frac{M-R^{P}}{R^{P}-L}\right)^{-\mathrm{RRA}} \lambda^{-1} \frac{H-M}{H-L}+\left(\frac{M-R^{P}}{H-R^{P}}\right)^{-\mathrm{RRA}} \frac{M-L}{H-L}-1$

Clearly, the condition $X^{P}=0$ implicitly defines $R^{P}$. For $R R A$ approaching $0, X^{P}$ approaches $\lambda^{-1} \frac{H-M}{H-L}+\frac{M-L}{H-L}-1$, which is smaller than zero given that $\lambda>1$. It follows that for $R R A$ approaching 0 , in the range of $R^{P}$ from $L$ to $M, R^{P}$ should be put as large as possible to achieve a minimum $\alpha$, so that $R^{P}$ approaches $M$. For RRA approaching $1, X^{P}$ approaches $\left(\frac{R^{P}-L}{M-R^{P}}\right)\left(\lambda^{-1}+1\right) \frac{H-M}{H-L}>0$. It follows that for RRA approaching 1 , in the range of $R^{P}$ from $L$ to $M, R^{P}$ should be put as small as possible to achieve a minimum $\alpha$, so that $R^{P}$ approaches $L$. This suggests that $\partial R^{P} / \partial \mathrm{RRA}<0$ for RRA from 0 to 1 , as we will show. Furthermore, for $R^{P}$ such that $X^{P}=0$, as we increase $\lambda, X^{P}$ becomes negative, suggesting that $R^{P}$ should be increased, as will also be shown.

Given that the denominator of (14) is positive, and given that $R=R^{P}$ when the numerator of (14) is zero iff $X^{P}=0$, we have $\left.\operatorname{sgn} \frac{\partial^{2} \alpha(L<R \leq M)}{\partial R^{2}}\right|_{R=R^{P}}=\operatorname{sgn} \frac{\partial X^{P}}{\partial R^{P}}$ (which is larger than zero by the second-order condition, as can indeed be checked). By the same reasoning, we have $\left.\operatorname{sgn} \frac{\partial^{2} \alpha(L<R \leq M)}{\partial R \partial \lambda}\right|_{R=R^{P}}=\operatorname{sgn} \frac{\partial X^{P}}{\partial \lambda}$. By (26), it follows that $\operatorname{sgn} \frac{\partial R^{P}}{\partial \lambda}=-\operatorname{sgn} \frac{\partial X^{P}}{\partial \lambda}$. Given that $\partial X^{P} / \partial \lambda<0$, it follows that $\partial R^{P} / \partial \lambda>0$. By the same reasoning, $\left.\operatorname{sgn} \frac{\partial^{2} \alpha(L<R \leq M)}{\partial R \partial \mathrm{RRA}}\right|_{R=R^{P}}=\operatorname{sgn} \frac{\partial X^{P}}{\partial \mathrm{RRA}}$. By (28), it follows that $\operatorname{sgn} \frac{\partial R^{P}}{\partial R R A}=-\operatorname{sgn} \frac{\partial X^{P}}{\partial R_{R A}}$. Now

$$
\begin{aligned}
\frac{\partial X^{P}}{\partial \mathrm{RRA}}= & -\left(\frac{M-R^{P}}{R^{P}-L}\right)^{-\mathrm{RRA}} \ln \left(\frac{M-R^{P}}{R^{P}-L}\right) \lambda^{-1} \frac{H-M}{H-L} \\
& -\left(\frac{M-R^{P}}{H-R^{P}}\right)^{-\mathrm{RRA}} \ln \left(\frac{M-R^{P}}{H-R^{P}}\right) \frac{M-L}{H-L}
\end{aligned}
$$

As $M-R^{P}<H-R^{P}$, the second term of the RHS of (29) is positive. Furthermore, for $R^{P}>(L+M) / 2$, the first term is positive as well, so that $\partial X^{P} / \partial \mathrm{RRA}>0$, meaning that $\partial R^{P} / \partial \mathrm{RRA}<0$. This includes the case where RRA approaches 0 , in which case by the above $R^{P}$ approaches $M$, so that $R^{P}>(L+M) / 2, \partial X^{P} / \partial \mathrm{RRA}>0$. Furthermore, taking the derivative of the RHS of (29) with respect to RRA, we obtain

$$
\begin{aligned}
\frac{\partial^{2} X^{P}}{\partial \mathrm{RRA}^{2}}= & \left(\frac{M-R^{P}}{R^{P}-L}\right)^{-\mathrm{RRA}}\left[\ln \left(\frac{M-R^{P}}{R^{P}-L}\right)\right]^{2} \lambda^{-1} \frac{H-M}{H-L} \\
& +\left(\frac{M-R^{P}}{H-R^{P}}\right)^{-\mathrm{RRA}}\left[\ln \left(\frac{M-R^{P}}{H-R^{P}}\right)\right]^{2} \frac{M-L}{H-L}>0
\end{aligned}
$$

Given that $\partial X^{P} / \partial$ RRA $>0$ for the lowest possible RRA, and given that $\partial X^{P} / \partial \mathrm{RRA}$ is everywhere increasing in RRA, it follows that it is everywhere the case that $\partial X^{P} / \partial \mathrm{RRA}>0$, meaning that $\partial R^{P} / \partial \mathrm{RRA}<0$. Indeed, for RRA approaching 1 , as already shown above, $R^{P}$ approaches $L$.

Step 3. By Proposition 1 II(c), for any CARA, any $R^{C}$ such that $H \leq R^{C} \leq+\infty$ is optimal. It follows that $R^{C}$ does not change a function of $\lambda$ or RRA. 
Step 4. The sign of $\partial R^{C} / \partial \lambda$ can be found by taking the total differential of $\left.\frac{\partial \alpha(M<R \leq H)}{\partial R}\right|_{R=R^{C}}=0$ with respect to $R^{C}$ and $\lambda$, and equating to zero, or

$$
\left.\frac{\partial^{2} \alpha(M<R \leq H)}{\partial R^{2}}\right|_{R=R^{C}} \mathrm{~d} R^{C}+\left.\frac{\partial^{2} \alpha(M<R \leq H)}{\partial R \partial \lambda}\right|_{R=R^{C}} \mathrm{~d} \lambda=0
$$

In the same way, the sign of $\partial R^{C} / \partial \mathrm{RRA}$ can be found by taking the total differential of $\left.\frac{\partial \alpha(M<R \leq H)}{\partial R}\right|_{R=R^{C}}=0$ with respect to $R^{P}$ and $R R A$, and equating to zero, or

$$
\left.\frac{\partial^{2} \alpha(M<R \leq H)}{\partial R^{2}}\right|_{R=R^{C}} \mathrm{~d} R^{C}+\left.\frac{\partial^{2} \alpha(M<R \leq H)}{\partial R \partial \mathrm{RRA}}\right|_{R=R^{C}} \mathrm{dRRA}=0
$$

Looking at (15), one obtains $\left.\frac{\partial \alpha(M<R \leq H)}{\partial R}\right|_{R=R^{C}}=0$ when the numerator of the RHS of (15) equals zero, which under the generic CRRA valuation function $v(X)=$ $X^{1-R R A}$ can be written as

$$
(1-\mathrm{RRA})\left(H-R^{C}\right)^{-\mathrm{RRA}} \lambda\left(R^{C}-M\right)^{-\mathrm{RRA}}(H-M)\left\{X^{C}\right\}=0
$$

where $X^{C}=\left(\frac{R^{C}-L}{H-R^{C}}\right)^{-\mathrm{RRA}} \lambda \frac{M-L}{H-M}-\left(\frac{R^{C}-L}{R^{C}-M}\right)^{-\mathrm{RRA}} \frac{H-L}{H-M}+1$

Clearly, the condition $X^{C}=0$ implicitly defines $R^{C}$. For RRA approaching 0 , $X^{C}$ approaches $\lambda \frac{M-L}{H-M}-\frac{H-L}{H-M}+1$, which is larger than zero. It follows that in the range of $R^{C}$ from $M$ to $H, R^{C}$ should be put as large as possible to achieve a maximum, so that $R^{C}$ approaches $H$. For RRA approaching 1, it can be checked that $X^{C}$ approaches $\left(\frac{H-R^{C}}{R^{C}-L}\right)(\lambda+1) \frac{M-L}{H-M}>0$. It follows that $R^{C}$ once more approaches $H$. This suggests that for low RRA we have $\partial R^{C} / \partial \mathrm{RRA}<0$, whereas for high RRA we have $\partial R^{C} / \partial \mathrm{RRA}>0$, which we now show. Furthermore, for $R^{C}$ such that $X^{C}=0$, as we increase $\lambda, X^{C}$ becomes positive, suggesting that $R^{C}$ should be increased, as will also be shown.

Given that the denominator of (15) is positive, and given that (32) is valid when $X^{C}=0$, we have $\left.\operatorname{sgn} \frac{\partial^{2} \alpha(M<R \leq H)}{\partial R^{2}}\right|_{R=R^{C}}=\operatorname{sgn} \frac{\partial X^{C}}{\partial R^{C}}$ (which is smaller than zero by the second-order condition, as can indeed be checked). In the same way, sgn $\left.\frac{\partial^{2} \alpha(M<R \leq H)}{\partial R \partial \lambda}\right|_{R=R^{C}}=\operatorname{sgn} \frac{\partial X^{C}}{\partial \lambda}$. By (30), it follows that $\operatorname{sgn} \frac{\partial R^{C}}{\partial \lambda}=\operatorname{sgn} \frac{\partial X^{C}}{\partial \lambda}$. Given that $\partial X^{C} / \partial \lambda>0$, it follows that $\partial R^{C} / \partial \lambda>0$. Furthermore, $\left.\operatorname{sgn} \frac{\partial^{2} \alpha(M<R \leq H)}{\partial R \partial \operatorname{RRA}}\right|_{R=R^{C}}=$ $\operatorname{sgn} \frac{\partial X^{C}}{\partial R R A}$. By (31), it follows that $\operatorname{sgn} \frac{\partial R^{C}}{\partial R R A}=\operatorname{sgn} \frac{\partial X^{C}}{\partial R R A}$. Now

$$
\begin{aligned}
\frac{\partial X^{C}}{\partial \mathrm{RRA}}= & -\left(\frac{R^{C}-L}{H-R^{C}}\right)^{-\mathrm{RRA}} \ln \left(\frac{R^{C}-L}{H-R^{C}}\right) \lambda \frac{M-L}{H-M} \\
& +\left(\frac{R^{C}-L}{R^{C}-M}\right)^{-\mathrm{RRA}} \ln \left(\frac{R^{C}-L}{R^{C}-M}\right) \frac{H-L}{H-M}
\end{aligned}
$$


As $R^{C}-L>R^{C}-M$, the second term of the RHS of (33) is positive. Furthermore, for $R^{C}>(L+H) / 2$, the first term of the RHS of (33) is negative, so that $\partial X^{C} / \partial \mathrm{RRA}$ and $\partial R^{C} / \partial \mathrm{RRA}$ may be ambiguous. Indeed, for RRA approaching 0 , as already shown, $R^{C}$ approaches $H$, in which case it can be checked that $\partial X^{C} / \partial \mathrm{RRA}$ is negative. Also, for RRA approaching $1, R^{C}$ again approaches $H$, in which case it can be checked that $\partial X^{C} / \partial \mathrm{RRA}$ is positive.

\section{Appendix 3}

\section{See Table 1}

Table 1 List of symbols

\begin{tabular}{|c|c|}
\hline$M$ & $\begin{array}{l}\text { Unique outcome of the safe action (= preventive treatment, failure to buy } \\
\text { curative treatment) }\end{array}$ \\
\hline$L(H)$ & $\begin{array}{l}\text { Low (high) outcome obtained from risky action (= failure to buy } \\
\text { preventive treatment, curative treatment) }\end{array}$ \\
\hline$X$ & Generic outcome $(M, L$, or $H)$ \\
\hline$p_{L}\left(p_{H}\right)$ & objective probability of the low (high) outcome \\
\hline$R$ & Reference point \\
\hline$R_{\min }\left(R_{\max }\right)$ & Minimal possible (maximal) reference point \\
\hline$X-R(R-X)$ & Gain for $X \geq R($ loss for $X<R)$ \\
\hline$\lambda$ & Degree of loss aversion \\
\hline$v(X-R)(-\lambda v(R-X))$ & Psychic valuation function for gains (losses) \\
\hline$f(\cdot)$ & Psychic valuation function $(=v(X-R)$ for gains, $=-\lambda v(R-X)$ for loss $)$ \\
\hline$\alpha$ & Measure of global risk tolerance $\left(\alpha=\frac{f(H)-f(M)}{f(M)-f(L)}\right)$ \\
\hline$o\left(p_{L}, p_{H}\right)($ in short $o$ ) & Odds of failure when taking risky action $\left(o\left(p_{L}, p_{H}\right)=\frac{p_{L}}{p_{H}}\right)$ \\
\hline$o_{\min }\left(o_{\max }\right)$ & Minimal (maximal) possible odds of failure \\
\hline$g(\cdot)$ & Density function of $o$ over $\left[o_{\min }, o_{\max }\right]$ \\
\hline$G[\cdot]$ & Cumulative distribution function of $o$ over $\left[o_{\min }, o_{\max }\right]$ \\
\hline$R^{C}\left(R^{P}\right)$ & $\begin{array}{l}\text { Reference point for which global risk tolerance } \alpha(R) \text { is maximized } \\
\text { (minimized), and optimal reference point in the curative (preventive } \\
\text { scenario) }\end{array}$ \\
\hline ARA & Absolute rate of risk aversion $\left(=-v^{\prime \prime}(\cdot) / v^{\prime}(\cdot)\right)$ \\
\hline RRA & $\begin{array}{l}\text { Relative rate of risk aversion }\left(=-Y v^{\prime \prime}(\cdot) / v^{\prime}(\cdot)\right) \text { (where } Y=(X-R) \text { in } \\
\text { case of gains, and } Y=(R-X) \text { in case of losses) }\end{array}$ \\
\hline DARA & Psychic valuation function where ARA decreases in the outcome \\
\hline CARA & Psychic valuation function where ARA is constant in the outcome \\
\hline CRRA & Psychic valuation function where RRA is constant in the outcome \\
\hline
\end{tabular}




\section{References}

Armantier, O., \& Boly, A. (2015). Framing of incentives and effort provision. International Economic Review, 56, 917-938.

Attema, A. E., Brouwer, W. B. F., \& L'Haridon, O. (2012). Prospect theory in the health domain: A quantitative assessment (Unpublished manuscript).

Azevedo, E. M., \& Gottlieb, D. (2012). Risk-neutral firms can extract unbounded profits from consumers with prospect theoretic preferences. Journal of Economic Theory, 147, 1291-1299.

Beck, A., Kerschbamer, R., Qiu, J., \& Sutter, M. (2013). Shaping beliefs in experimental markets for expert services: Guilt aversion and the impact of promises and money-burning options. Games and Economic Behavior, 81, 145-164.

Booij, A. S., van Praag, B. M. S., \& van de Kuilen, G. (2010). A parametric analysis of prospect theory's functionals for the general population. Theory and Decision, 68, 115-148.

Calcott, P. (1999). Demand inducement as cheap talk. Health Economics, 8, 721-733.

Darby, M. R., \& Karni, R. (1973). Free competition and the optimal amount of fraud. Journal of Law and Economics, 16, 67-88.

De Jaegher, K., \& Jegers, M. (2001). The physician-patient relationship as a game of strategic information transmission. Health Economics, 10, 651-668.

Dranove, D. (1988). Demand inducement and the physician/patient relationship. Economic Inquiry, 26, 281-298.

Dulleck, U., \& Kerschbamer, R. (2006). On doctors, mechanics, and computer specialists: The economics of credence goods. Journal of Economic Literature, 44, 5-42.

Evans, R. G. (1974). Supplier-induced demand: some empirical evidence and implications. In M. Perlman (Ed.), The economics of health and medical care (pp. 162-173). London: Macmillan.

Goerg, S.J., \& Kube, S. (2012). Goals (th)at work-goals, monetary incentives, and workers' performance. Preprints of the Max Planck Institute for Research on Collective Goods, University of Bonn, No. 2012/19.

Halpern, J. Y., \& Rêgo, L. C. (2007). Extensive games with possibly unaware players. Mathematical Social Sciences, 70, 42-58.

Hong, H., Hossain, T., \& List, J. A. (2015). Framing manipulations in contests: A natural field experiment. Journal of Economic Behavior and Organization, 118, 372-382.

Hossain, T., \& List, J. A. (2012). The behavioralist visits the factory: Increasing productivity using simple framing manipulations. Management Science, 58, 2151-2167.

Kahneman, D. (1999). Objective happiness. In D. Kahneman, E. Diener, \& N. Schwarz (Eds.), Well-being: The foundations of hedonic psychology (pp. 3-25). New York: Russell Sage Foundation.

Kahneman, D., \& Tversky, A. (1979). Prospect theory: An analysis of decision under risk. Econometrica, 47, 263-291.

Kahneman, D., Wakker, P., \& Sarin, R. (1997). Back to Bentham? Explorations of experienced utility. Quarterly Journal of Economics, 112, 375-405.

Kamenica, E., \& Gentzkow, M. (2011). Bayesian persuasion. American Economic Review, 101, 2590-2615.

Kerschbamer, R., Sutter, M., \& Dulleck, U. (2017). How social preferences shape incentives in (experimental) markets for credence goods. Economic Journal, 127, 393-416.

Kőszegi, B., \& Rabin, M. (2006). A model of reference-dependent preferences. Quarterly Journal of Economics, 121, 1133-1165.

Kühberger, A. (1998). The influence of framing on risky decisions: A meta-analysis. Organizational Behavior and Human Decision Processes, 75, 23-55.

Loewenstein, G. (2005). Hot-cold empathy gaps and medical decision making. Health Psychology, 24, 849-856.

Norton, D. A., \& Isaac, M. A. (2012). Experts with a conflict of interest: A source of ambiguity? Experimental Economics, 15, 260-277.

Page, L. (2005). Des inégalités sociales aux inégalités scolaires-choix éducatifs et prospect theory. Revue Économique, 56, 615-623.

Peacock, S., \& Richardson, J. (2007). Supplier-induced demand re-examining identification and misspecification in cross-sectional analysis. European Journal of Health Economics, 8, 267-277.

Pesendorfer, W., \& Wolinsky, A. (2003). Second opinions and price competition: Inefficiency in the market for expert advice. Review of Economics Studies, 70, 417-437. 
Pitchik, C., \& Schotter, A. (1987). Honesty in a model of strategic information transmission. American Economic Review, 77, 1032-1036.

Pope, D. G., \& Schweitzer, M. E. (2011). Is Tiger Woods loss averse? Persistent bias in the face of experience, competition, and high stakes. American Economic Review, 101, 129-157.

Puppe, C., \& Rosenkranz, S. (2011). Why suggest non-binding retail prices? Economica, 78, 317-329.

Rosenkranz, S., \& Schmitz, P. (2007). Reserve prices in auctions as reference points. Economic Journal, 117, 637-653.

Rothman, A. J., Bartels, R. D., Wlaschin, J., \& Salovey, P. (2006). The strategic use of gain- and loss-framed messages to promote healthy behaviour: How theory can inform practice. Journal of Communication, 56, S202-S220.

Rothman, A. J., \& Salovey, P. (1997). Shaping perceptions to motivate healthy behaviour: The role of message framing. Psychological Bulletin, 121, 3-19.

Salant, Y., \& Siegel, L. (2018). Contracts with framing. American Economic Journal Microeconomics, 10, 315-346.

Schwartz, A., Goldberg, J., \& Hazen, G. (2008). Prospect theory, reference points, and health decisions. Judgment and Decision Making, 3, 174-180.

Spiegler, R. (2006). The market for quacks. Review of Economic Studies, 73, 1113-1131.

Taylor, C. R. (1995). The economics of breakdowns, checkups, and cures. Journal of Political Economy, 103, 53-74.

Thaler, R. H. (1999). Mental accounting matters. Journal of Behavioral Decision Making, 12, 183-206.

Tversky, A., \& Kahneman, D. (1981). The framing of decisions and the psychology of choice. Science, 211, $453-458$.

Tversky, A., \& Kahneman, D. (1992). Advances in prospect theory: Cumulative representation of uncertainty. Journal of Risk and Uncertainty, 5, 297-323.

van der Pol, M., \& Ruggeri, M. (2008). Is risk attitude outcome specific within the health domain? Journal of Health Economics, 27, 706-717.

Wakker, P. P. (2010). Prospect Theory for Risk and Ambiguity. Cambridge: Cambridge University Press.

Publisher's Note Springer Nature remains neutral with regard to jurisdictional claims in published maps and institutional affiliations. 